\title{
Nanoparticle-Mediated Targeting of Pitavastatin to Small Pulmonary Arteries and Leukocytes by Intravenous Administration Attenuates the Progression of Monocrotaline-Induced Established Pulmonary Arterial Hypertension in Rats
}

Kenzo Ichimura, ${ }^{1} \mathrm{MD}$, Tetsuya Matoba, ${ }^{1} \mathrm{MD}$, Jun-ichiro Koga, ${ }^{2} \mathrm{MD}$, Kaku Nakano, ${ }^{2} \mathrm{PhD}$, Daiki Funamoto, ${ }^{2} \mathrm{PhD}$, Hiroyuki Tsutsui, ${ }^{1} \mathrm{MD}$ and Kensuke Egashira, ${ }^{2} \mathrm{MD}$

\begin{abstract}
Summary
Statins are known to improve pulmonary arterial hypertension (PAH) by their anti-inflammatory and antiproliferative effects in animal models. However, recent clinical studies have reported that clinically approved statin doses failed to improve clinical outcomes in patients with PAH. We therefore hypothesized that nanoparticle (NP)-mediated targeting of pitavastatin could attenuate the progression of established PAH.

We induced PAH by subcutaneously injecting monocrotaline (MCT) in Sprague-Dawley rats. On day 14 after the MCT injection, animals that displayed established PAH on echocardiography were included. On day 17, they were randomly assigned to the following 5 groups: daily intravenous administration of (1) vehicle, (2) fluorescein-isothiocyanate-NP, (3) pitavastatin, (4) pitavastatin-NP, or (5) oral sildenafil. Intravenous NP was selectively delivered to small pulmonary arteries and circulating CD11b-positive leukocytes. On day 21, pitavastatin-NP attenuated the progression of PAH at lower doses than pitavastatin alone. This was associated with the inhibition of monocyte-mediated inflammation, proliferation, and remodeling of the pulmonary arteries. Interestingly, sildenafil attenuated the development of PAH, but had no effects on inflammation or remodeling of the pulmonary arteries. In separate experiments, only treatment with pitavastatin-NP reduced the mortality rate at day 35.

NP-mediated targeting of pitavastatin to small pulmonary arteries and leukocytes attenuated the progression of established MCT-induced PAH and improved survival. Therapeutically, pitavastatin-NP was associated with anti-inflammatory and anti-proliferative effects on small pulmonary arteries, which was completely distinct from the vasodilatory effect of sildenafil. Pitavastatin-NP can be a novel therapeutic modality for PAH.
\end{abstract}

(Int Heart J 2018; 59: 1432-1444)

Key words: Translational study, Statin, Pulmonary hypertension

$\mathrm{P}$ ulmonary arterial hypertension (PAH) is an intractable disease of the small pulmonary arteries that results in a progressive increase in pulmonary vascular resistance and ultimately leads to right ventricular (RV) failure and premature death. A recent registry of patients with $\mathrm{PAH}$ revealed that mortality from $\mathrm{PAH}$ remains high, even after the introduction of several vasodilator therapies. ${ }^{1)}$ Therefore, a novel therapy to improve mortality is warranted.

PAH has a complex and multifactorial pathogenesis, characterized by excessive inflammation and proliferation of pulmonary vascular cells, namely, endothelial cells and smooth muscle cells (SMCs), which heavily contribute to inappropriate pulmonary vascular remodeling. ${ }^{2}$ The 3hydroxy-3-methylglutaryl coenzyme-A reductase inhibitors (statins) inhibit the isoprenylation of small GTPases, such as Ras, Rho, and Rac, which are well-known key mediators of cellular proliferation, ${ }^{3)}$ endothelial nitric oxide synthase (eNOS) expression, ${ }^{4}$ inflammation, ${ }^{5}$ and oxidative stress $^{(6)}$ in the vascular wall cells. ${ }^{7,8)}$ These pleiotropic effects of statins have led to the rationale of utilizing them for the treatment of PAH. ${ }^{9)}$ Indeed, prior animal studies have revealed that the systemic administration of statins at high doses attenuated PAH progression in several rodent

From the ${ }^{1}$ Department of Cardiovascular Medicine, Kyushu University Graduate School of Medical Sciences, Fukuoka, Japan and ${ }^{2}$ Department of Cardiovascular Research, Development, and Translational Medicine, Center for Disruptive Cardiovascular Medicine, Kyushu University, Fukuoka, Japan.

This study was supported by grants from the Ministry of Education, Science, and Culture, Tokyo, Japan (Grants-in-Aid for Scientific Research 25461135 to Matoba and 23790863 and 23790861 to Egashira) and the Intractable diseases overcome research project from Japan Agency for Medical Research and development, AMED (to Egashira).

Address for correspondence: Kensuke Egashira, MD, Department of Cardiovascular Research, Development, and Translational Medicine, Center for Disruptive Cardiovascular Medicine, Kyushu University, 3-1-1 Maidashi, Higashi-ku, Fukuoka 812-8582, Japan. E-mail: egashira@cardiol.med.kyushu-u.ac.jp

Received for publication November 30, 2017. Revised and accepted January 16, 2018.

Released in advance online on J-STAGE October 25, 2018

doi: 10.1536/ihj.17-683

All rights reserved by the International Heart Journal Association. 


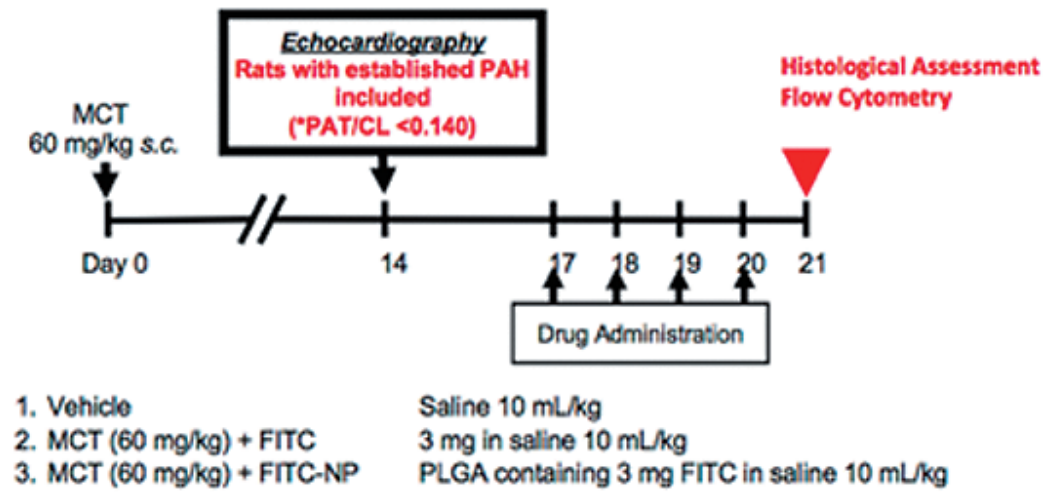

Figure 1. Experimental protocols for trace study. Scheme showing the experimental protocol for the trace study of FITC-NP.

models, which was associated with enhanced eNOS expression, ${ }^{9,10)}$ activation of eNOS, ${ }^{11)}$ and reduction of Rhokinase expression in the lungs. ${ }^{12,13)}$ However, recent clinical trials that tested the effect of statins on PAH with currently approved doses (atorvastatin $10 \mathrm{mg} /$ day or simvastatin $80 \mathrm{mg} /$ day) showed neutral results with regard to the clinical benefits for patients with $\mathrm{PAH}{ }^{14,15)}$ These results suggested that the currently approved doses of statins were not sufficient to exert beneficial effects on PAH and that a modality to enhance their efficacy is warranted. Therefore, a drug delivery system (DDS) that facilitates the targeting of statins to the lung would have greater clinical implications.

Recently, we developed a bioabsorbable poly-lactic/ glycolic acid (PLGA)-nanoparticle (NP)-mediated DDS for pitavastatin ${ }^{16-22)}$ and showed that direct intratracheal instillation of pitavastatin-NP attenuated the development of $\mathrm{PAH}$, with reductions in inflammation and pulmonary artery remodeling in a rat model of monocrotaline (MCT)induced $\mathrm{PAH}^{20)}$ Additionally, we reported that intravenously administered pitavastatin-NP was selectively delivered to tissues with enhanced vascular permeability by passive targeting and to tissues with inflammation by direct incorporation and delivery by circulating monocytes in a mouse model of atherosclerosis ${ }^{18,23}$ and myocardial infarction, ${ }^{24)}$ and also in rat and porcine models of myocardial ischemia-reperfusion injury. ${ }^{16,17)}$ Therefore, we hypothesized that intravenously administered pitavastatin-NP could be selectively delivered to small pulmonary arteries and inflammatory cells in the lung and exert its antiinflammatory and anti-proliferative effects on PAH. In the present study, we tested the efficacy of intravenous administration of pitavastatin-NP in a preclinical rat model of $\mathrm{PAH}$ to verify our hypothesis and translate the therapeutic effect of pitavastatin-NP into clinical practice.

\section{Methods}

Preparation of PLGA nanoparticles: PLGA nanoparticles incorporating fluorescein-isothiocyanate (FITC; Dojin Chemical, Tokyo) (FITC-NP) or pitavastatin (Kowa Pharmaceutical Co. Ltd., Tokyo) (pitavastatin-NP) were pre- pared by an emulsion solvent diffusion method as described previously. ${ }^{16-23,25-31)}$

Experimental animal models and protocols: All experiments were reviewed and approved by the Committee of Ethics of Animal Experiments, Kyushu University Graduate School of Medical Sciences. Male Sprague-Dawley (SD) rats (Charles River, Yokohama; weight 200-230 g) were subcutaneously injected with $60 \mathrm{mg} / \mathrm{kg}$ of MCT (Sigma-Aldrich, MO), which induces severe $\mathrm{PAH}$ in 3 weeks. ${ }^{20)}$ Pulmonary artery acceleration time/cycle length (PAAT/CL) was measured by echocardiography (Vevo 2100 ultrasound system; Visual Sonic, Toronto) in each animal at day 14 after the MCT injection and was used as a noninvasive estimation of pulmonary artery systolic pressure. ${ }^{32)}$ Animals with PAAT/CL $<0.140$ were assumed to have developed PAH and were included in the study. Experimental protocols are shown in Figures 1-3.

Echocardiographic measurement of RV and pulmonary artery hemodynamics: Transthoracic 2-D and pulsed-wave Doppler echo were obtained with a $30-\mathrm{MHz}$ transducer. Echocardiographic images were obtained as described previously. ${ }^{20)}$ Pulsed-wave Doppler was used to measure PAAT. During echocardiography, the animals were anesthetized with isoflurane (1\%, AbbVie, IL).

Flow cytometry of blood, lung, and bronchiolar alveolar lavage fluid (BALF): Peripheral blood was lysed with VersaLyse lysing solution (Becton Dickinson Bioscience, CA) for 15 minutes at room temperature. The lungs were isolated and digested with a cocktail of $450 \mathrm{U} / \mathrm{mL}$ collagenase type I, $125 \mathrm{U} / \mathrm{mL}$ collagenase type XI, $50 \mathrm{U} / \mathrm{mL}$ DNase I, and $60 \mathrm{U} / \mathrm{mL}$ hyaluronidase (Sigma-Aldrich) in phosphate-buffered saline (PBS) containing $20 \mathrm{mM}$ Hepes at $37^{\circ} \mathrm{C}$. BALF was collected in cold PBS. The cell suspension was centrifuged at $300 \times \mathrm{g}$ for 5 minutes at $4^{\circ} \mathrm{C}$. After blocking the Fc receptor with anti-CD32 antibody (Becton Dickinson Bioscience), cell suspensions were incubated with anti-CD11b antibody (Becton Dickinson Bioscience) or an isotype control for 1 hour at $4^{\circ} \mathrm{C}$. Finally, they were incubated with 7-AAD (Becton Dickinson Bioscience) for 5 minutes at room temperature and analyzed with Gallios (Beckman Coulter, CA).

Direct hemodynamic measurement: The animals were 


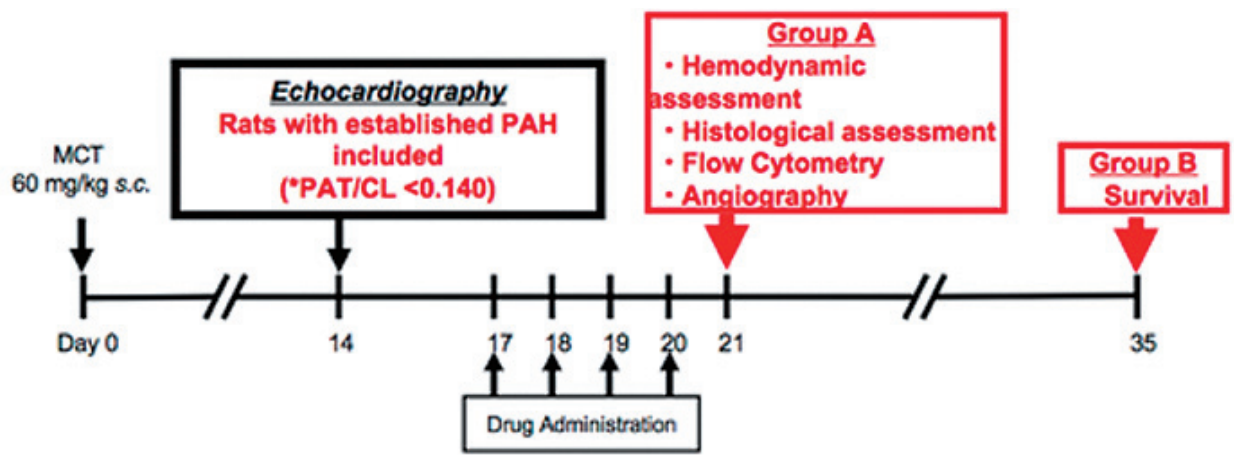

Group A

1. MCT $(60 \mathrm{mg} / \mathrm{kg})+$ Vehicle

2. NCT $(60 \mathrm{mg} / \mathrm{kg})+$ FITC-NP

3. NCT $(60 \mathrm{mg} / \mathrm{kg})+$ Pitavastatin

4. MCT $(60 \mathrm{mg} / \mathrm{kg})+$ Pitavastatin-NP

5. MCT $(60 \mathrm{mg} / \mathrm{kg})+$ Sildenafil

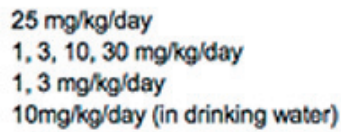

Group B

1. MCT $(60 \mathrm{mg} / \mathrm{kg})+$ Vehide

2. MCT $(60 \mathrm{mg} / \mathrm{kg})+$ FITC-NP

3. MCT $(60 \mathrm{mg} / \mathrm{kg})+$ Pitavastatin-NP

4. MCT $(60 \mathrm{mg} / \mathrm{kg})+$ Sildenafil

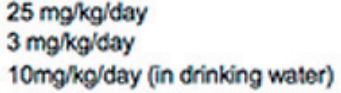

Figure 2. Experimental protocol for treatment. Scheme showing the experimental protocol for treatment.

anesthetized with isoflurane (1\%), and polyethylene catheters (PE10, Becton Dickinson, NJ; internal diameter, 0.28 $\mathrm{mm}$ ) were inserted into the right ventricle through the right jugular vein as described previously. ${ }^{3)} \mathrm{RV}$ systolic pressure (RVSP) was measured with a polygraph system (PowerLab, AD Instruments, Dunedin, New Zealand).

Assessment of RV hypertrophy: The RV wall was dissected from the left ventricle and the ventricular septum. The right and left ventricles plus the ventricular septum were weighed, and the degree of right ventricular hypertrophy (RVH) was quantified as RV weight/[left ventricle plus ventricular septum weights].

Histopathological and immunohistochemical analyses of the lungs: For paraffin sections, lungs were inflated with $1 \%$ formalin plus $0.5 \%$ agarose at $20 \mathrm{~cm} \mathrm{H}_{2} \mathrm{O}$ pressure and fixed in $10 \%$ formalin overnight. Then, they were embedded in paraffin and cut into 5- $\mu \mathrm{m}$ sections. Sections were incubated overnight at $4^{\circ} \mathrm{C}$ with the following primary antibodies: anti- $\alpha$-smooth muscle actin (SMA) antibody (dilution 1:500; clone 1A4, Dako, MI), anti-CD68 antibody (dilution 1:100; AbD Serotec, NC), anti-NF- $\kappa \mathrm{B}$ antibody (dilution 1:1,000; p65 subunit, clone 12H11, Merck Millipore, MA), and anti-PCNA antibody (dilution 1:400; Dako), followed by incubation with biotin-conjugated secondary antibodies.

The degree of remodeling in small peripheral pulmonary arteries was assessed by counting the number of SMA-positive pulmonary arteries by a blinded observer, as described previously. ${ }^{20}$ To quantify the degrees of monocyte infiltration and $\mathrm{NF}-\kappa \mathrm{B}$ activity in the lung, a blinded observer counted the number of ED-1-positive or
NF- $\kappa$ B-positive cells in 5 high-power fields. To quantify the degree of vascular cellular proliferation, a blinded observer counted the number of PCNA-positive cells in 10 intra-acinar arteries with diameters of 25-50 $\mu \mathrm{m}$ in each rat.

For OCT-embedded sections, the lungs were inflated with a mixture of 1:1 OCT compound (Sakura Finetechnical Co. Ltd., Tokyo) and PBS at $20 \mathrm{~cm} \mathrm{H}_{2} \mathrm{O}$ pressure and embedded in OCT compound at $-80^{\circ} \mathrm{C}$. Sections of 10 $\mu \mathrm{m}$ were cut and incubated with the following antibodies: anti-SMA antibody (dilution 1:200; Thermo Scientific, MA) and anti-von Willebrand factor (vWF) antibody (dilution 1:3,000, Dako), followed by incubation with Alexa Fluor 555-conjugated secondary antibody (anti-rabbit IgG Alexa Fluor 555 antibody, dilution 1:1,000, Invitrogen, MA). The nuclei were counterstained with DAPI. To quantify the fluorescence intensity of FITC, the mean fluorescence intensity of 20 randomly selected high-power fields was measured by ImageJ.

Ex-vivo angiography of pulmonary artery by Microfil: Fasudil hydrochloride hydrate $(10 \mathrm{mg} / \mathrm{kg}$ ) (Eril; Asahi Kasei Pharmaceutical Corporation, Tokyo) and heparin (100 units/100 g body weight) were injected into the femoral vein. Diluted heparin sodium ( 5 units/ $\mathrm{mL}, 20$ $\mathrm{mL}$ ) was injected via the right ventricle, and the heart and lungs were extracted. Polyethylene tubing (PE50, Becton Dickinson; internal diameter, $0.53 \mathrm{~mm}$ ) was advanced to the main pulmonary artery and secured in place using 5-0 silk sutures. A small incision was made in the left atrial appendage, and $2 \mathrm{~mL}$ of diluted heparin sodium was pumped at $2 \mathrm{~mL} / \mathrm{minute}$ using a continuous syringe pump 


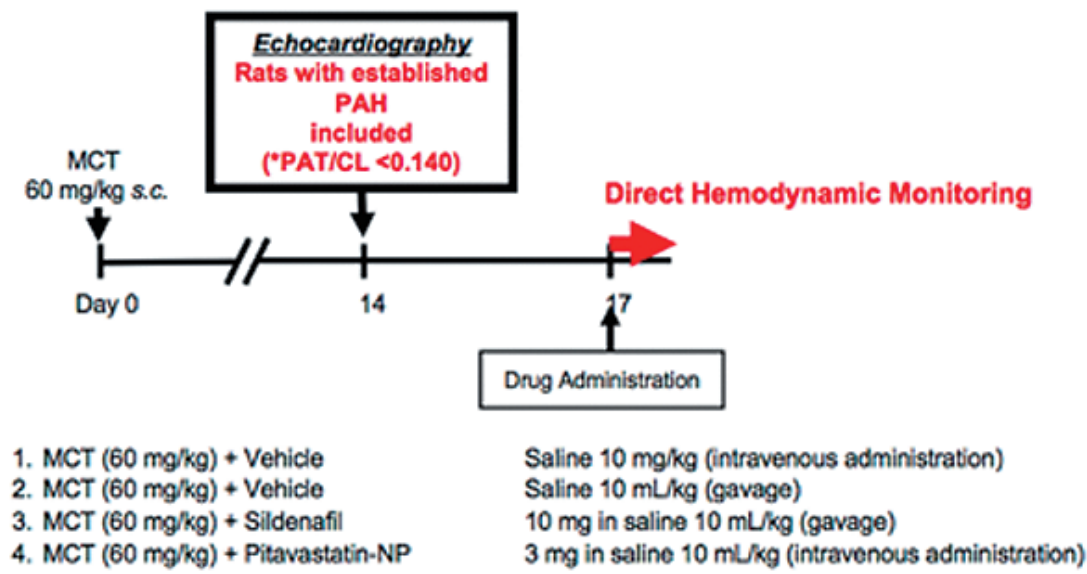

Figure 3. Experimental protocols for acute hemodynamic monitoring. Scheme showing the experimental protocol for acute hemodynamic monitoring.

(Melquest, Toyama). A silicone polymer casting compound, MV-yellow Microfil (Flow Tech, MA), was mixed with a medium-viscosity diluent in a 5:4 (diluent:compound) volume ratio and added to $5 \%$ (by volume) of the curing agent. This mixed silicone polymer casting material was pumped through the catheter at $0.1 \mathrm{~mL} / \mathrm{minute}$ until the polymer was uniformly visible on the lung surface. ${ }^{33)}$ The lungs were inflated with $1 \%$ formalin plus $0.5 \%$ agarose at $20 \mathrm{~cm} \mathrm{H}_{2} \mathrm{O}$ pressure. After complete polymerization at $4^{\circ} \mathrm{C}$ for 24 hours, the heart and lungs were fixed in $10 \%$ formalin. The lungs were sequentially bathed in serial concentrations of ethanol. The tissues were then placed in methyl salicylate and photographed with a stereoscopic microscope. ${ }^{34)}$

Measurements of cytokine, chemokine, and growth factor levels in the lung by multiplex immunoassay: $\mathrm{Cy}$ tokine, chemokine, and growth factor levels in the lung were measured with a cytokine magnetic-based assay: Bio-Plex Pro Rat Cytokine 24-Plex Panel (Bio-Rad, CA), according to the manufacturer's instructions. A heat map showing the means of the $\mathrm{z}$-scores $(\mathrm{z}=(\mathrm{x}-\mathrm{mean}) / \mathrm{SD})$ was used only to show the trend of cytokine/chemokine and growth factor expressions in each group.

Statistical analysis: The data are presented as the means \pm standard deviation (SD) and were analyzed using the Prism Software program (Graph Pad Software, San Diego, CA). The statistical analysis of differences in 2 groups was performed using the t-test. The statistical analysis of differences between more than 3 groups was performed using the one-way analysis of variance (ANOVA) followed by Dunnett's tests. The bar graphs in Figure 5B and $9 \mathrm{C}$ were compared using two-way ANOVA, followed by Tukey's multiple comparison tests. The survival curves were described with the Kaplan-Meier method, and the survival rates between the treatment groups were compared with the log-rank test. $P$ values $<0.05$ were considered statistically significant.

\section{Results}

Localization of intravenously administered FITC-NP in the lung and CD-11b-positive leukocytes in rats with MCT-induced PAH: We evaluated the localization of NP in the lung after the daily administration of FITC or FITC-NP in both MCT-induced PAH rats and normal rats. In MCT-induced PAH rats, strong FITC signals were observed in the vascular walls of the SMA-positive vessels and the bronchial epithelium in FITC-NP-injected animals, but only faint signals were detected in FITC-aloneinjected animals (Figure 4). FITC signals co-localized with SMA (Figure 5), but not with vWF (Figure 6). In normal rats, less significant FITC signals were observed in FITC or FITC-NP-injected animals (Figures 4, 5).

Flow cytometry showed that animals treated with FITC-NP had significantly stronger FITC signals in CD11 b-positive leukocytes in the blood, lung, and BALF than those treated with FITC alone (Figure 7).

Effects of pitavastatin-NP on the development of PAH in the rat model of MCT-induced PAH: MCT injections induced severe PAH, which was characterized by elevated RVSP (Figure 8A), development of RVH (Figure 8B), and pulmonary artery remodeling (Figure 8C, D). On day 14, PAAT/CL measured using echocardiography was $>0.140$ in 9 of 108 animals, indicating low pulmonary artery systolic pressure, and these animals were excluded. On day 17, the included 99 animals were randomized into 4 groups and received intravenous treatment with vehicle, FITC-NP, pitavastatin alone, or pitavastatin-NP in several doses. Treatment with pitavastatin-NP containing 1 or 3 $\mathrm{mg} / \mathrm{kg}$ of pitavastatin significantly attenuated the elevation of RVSP, the development of RVH, and pulmonary artery remodeling compared with the control nanoparticle treatment (FITC-NP) and with the same dose of pitavastatin alone. Treatment with pitavastatin alone at the dose of 30 $\mathrm{mg} / \mathrm{kg}$, but not at doses of 1,3 , or $10 \mathrm{mg} / \mathrm{kg}$, also attenuated the development of RVH and pulmonary artery remodeling compared with vehicle treatment. These data 


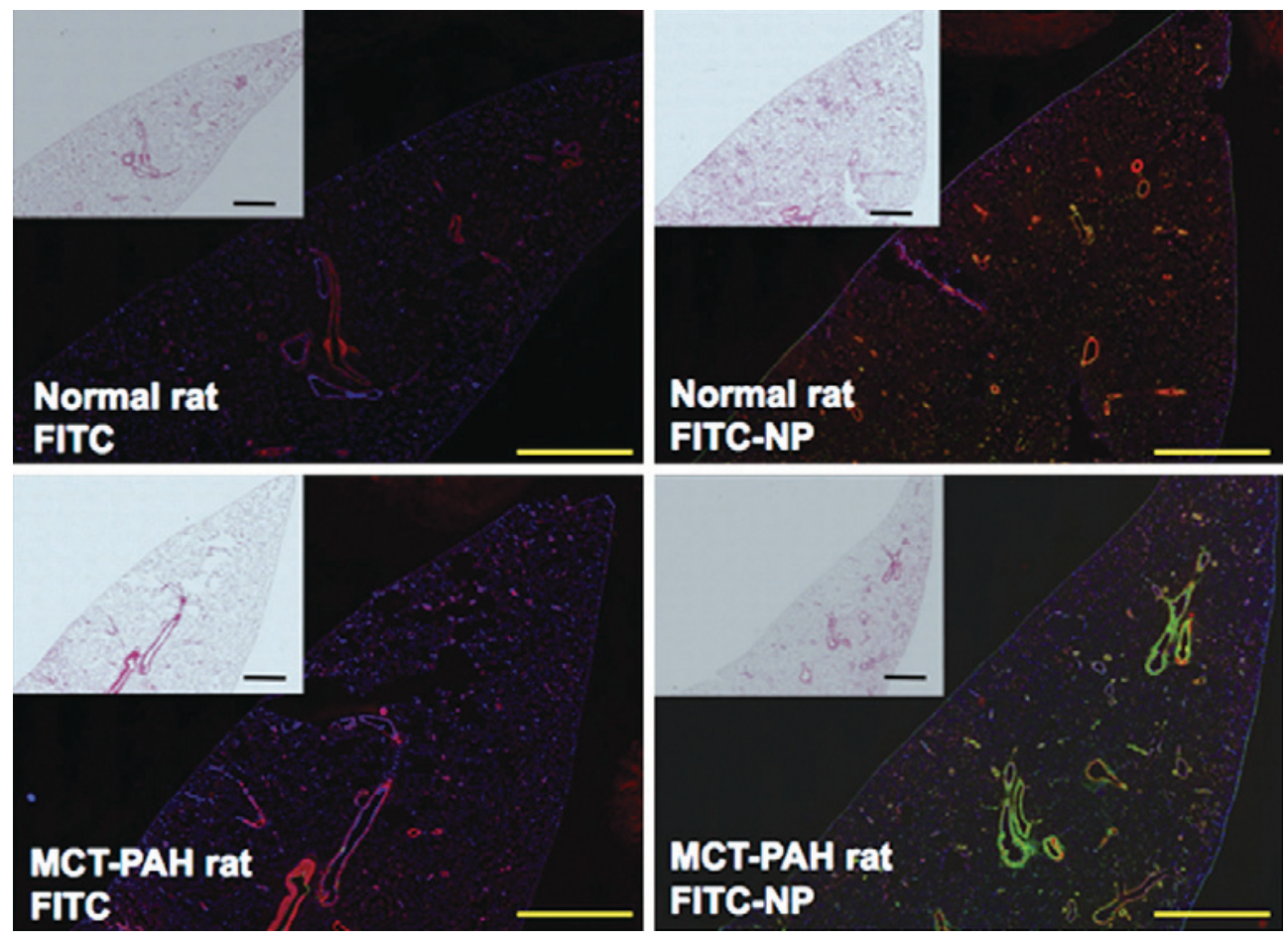

Figure 4. Delivery of FITC-NP to the lung. Representative bright field and fluorescence microscopic views of lung sections in normal rats or MCT-induced PAH rats after intravenous injection of FITC or FITC-NP. Fluorescence views are immunostained with smooth muscle actin (SMA; red). The nuclei are counterstained with DAPI. Scale bar: $1 \mathrm{~cm}$.

A

FITC

SMA

Merge
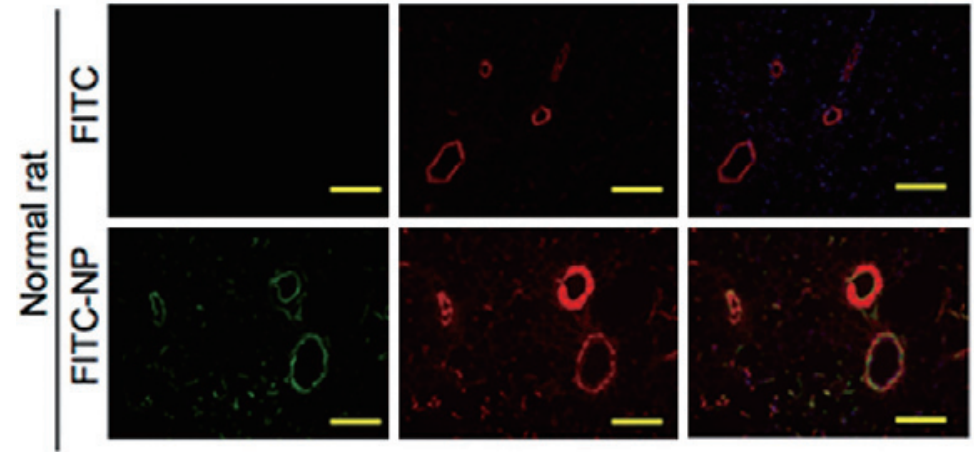

B
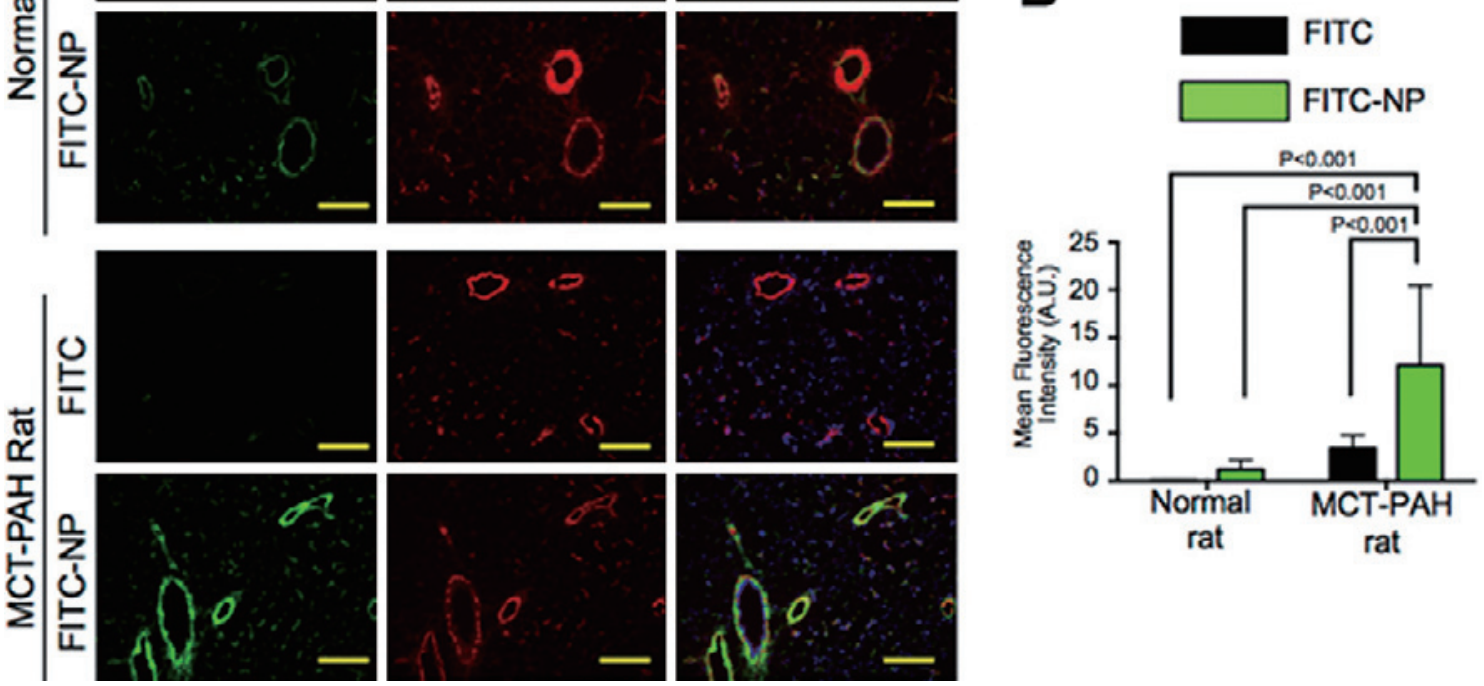

Figure 5. Magnified view of Figure 4. A: Representative magnified view of lung sections. Scale bar: $100 \mu \mathrm{m}$. B: Bar graph showing the quantitative measurement of FITC fluorescence intensity of lung sections shown in A. $n=20$ each 


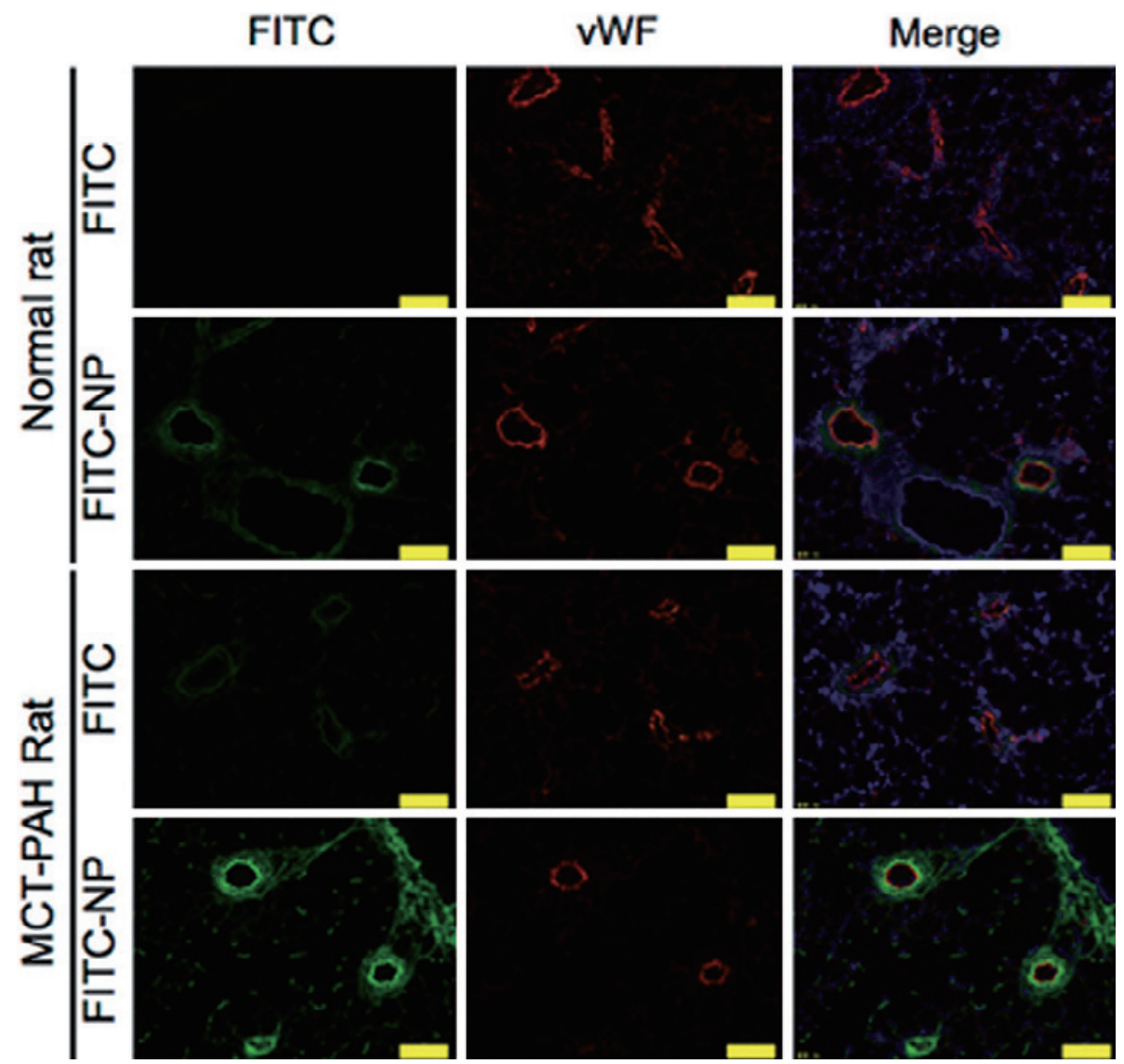

Figure 6. Immunohistochemistry of the lung with von Willebrand factor (vWF) after the daily intravenous administration of FITC or FITC-NP. Representative magnified view of lung sections. Scale bar: $100 \mu \mathrm{m}$.

suggested that pitavastatin-NP had approximately 30 times higher efficacy than pitavastatin alone in attenuating the development of PAH induced by the MCT injection.

We also performed ex-vivo angiography of the pulmonary arteries with Microfil to assess the effect of pitavastatin-NP on the development of small pulmonary artery stenosis and obstruction 3 weeks after the MCT injection (Figure 9A). The degree of small pulmonary artery stenosis and obstruction was quantified by counting the number of small vessels branching from the 2 nd, $3 \mathrm{rd}$, or 4 th branch of the main pulmonary artery (Figure 9B). The number of patent vessels branching from the $3 \mathrm{rd}$ or 4 th branch was significantly reduced in the vehicle and pitavastatin-alone $(3 \mathrm{mg} / \mathrm{kg})$ groups, but it was preserved in the pitavastatin-NP (3 $\mathrm{mg} / \mathrm{kg}$ of pitavastatin) group (Figure 9C).

Effects of pitavastatin-NP on inflammation in rats with MCT-induced PAH: Treatment with pitavastatin-NP containing 1 or $3 \mathrm{mg} / \mathrm{kg}$ of pitavastatin significantly attenuated the infiltration of CD68-positive monocytes, and the increase in NF- $\mathrm{KB}$ activity induced by MCT injection compared with the control nanoparticle treatment (FITCNP) and with the same dose of pitavastatin alone. Also, treatment with pitavastatin alone at the dose of $30 \mathrm{mg} / \mathrm{kg}$, but not at doses of 1,3 , or $10 \mathrm{mg} / \mathrm{kg}$, also attenuated the increase in $\mathrm{NF}-\kappa \mathrm{B}$ activity compared with vehicle treatment (Figure 10A, B). These data suggested that NPmediated targeting of pitavastatin to the lung by intravenous injection is more effective in blocking the inflammatory pathway and monocyte infiltration than pitavastatin alone.

We also performed a multiplex immunoassay to measure cytokines, chemokines, and growth factors simultaneously. The MCT injection induced a significant elevation in the cytokine/chemokine levels of the lung associated with monocytes/macrophages, such as MCP-1, MIP-1 $\alpha, \mathrm{GRO} / \mathrm{KC}$, and IL- $1 \alpha$. On the other hand, the MCT injection suppressed cytokines/chemokines associated with lymphocytes, such as IL-2, IL-4, IL-7, IL-10, IL-18, and IFN- $\gamma$. Pitavastatin-NP attenuated the elevation of monocyte/macrophage-associated cytokines/chemokines, such as MIP-1 $\alpha$, MIP-3 $\alpha$, GRO/KC, M-CSF, and IL- $1 \alpha$, compared to the vehicle, but it had no influence on lymphocyte-associated cytokines/chemokines (Figure 11). Comparison of the effects of pitavastatin-NP with PDE 5 inhibitor sildenafil on MCT-induced PAH: To compare the effects of pitavastatin-NP with the currently approved vasodilatory therapy, we administered the PDE5 


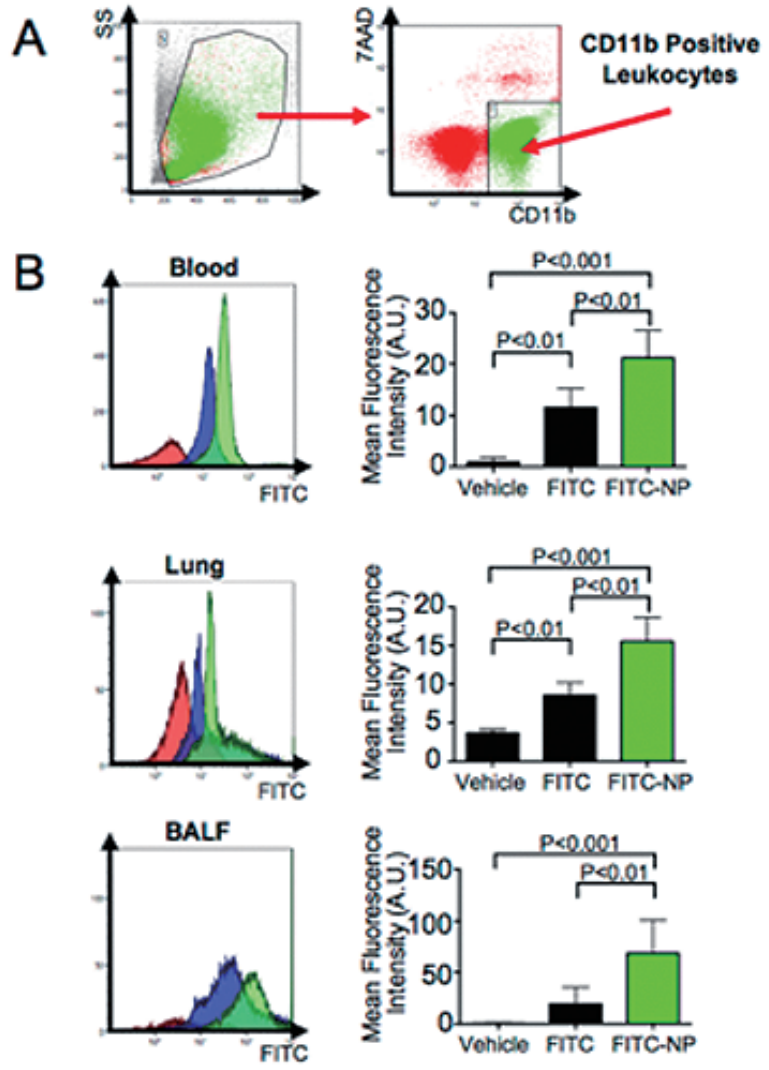

Figure 7. Flow cytometry of the blood, lung, and BALF in MCTinduced PAH rats. A: Gating strategy for CD11b-positive leukocytes. B: Representative histograms of CD11b-positive leukocytes showing FITC intensity in the blood, lung, and BALF after intravenous injections of vehicle, FITC, or FITC-NP. Red, blue, and green histograms indicate vehicle-, FITC-, and FITC-NP-treated groups, respectively. The bar graph shows the mean fluorescence intensity in CD11b-positive leukocytes. $n=5$ each.

inhibitor, sildenafil, via drinking water in the same treatment protocol as pitavastatin-NP (Figure 2). Orally administered sildenafil $(10 \mathrm{mg} / \mathrm{kg} / \mathrm{day})$ attenuated the elevation of RVSP and RVH comparable with pitavastatin-NP (Figure $12 \mathrm{~A}, \mathrm{~B})$. However, sildenafil failed to attenuate pulmonary artery remodeling in our treatment protocol (Figure 12C). Additionally, it did not reduce the inflammation in the lung (Figure 12D, E). Immunohistochemistry of the lung with PCNA also confirmed that pitavastatin-NP significantly reduced the proliferation of the vascular cells compared with vehicle treatment, but sildenafil did not (Figure 12F).

To examine the acute vasodilatory properties of pitavastatin-NP and sildenafil, we administered these drugs under continuous monitoring of RV and arterial pressures. In contrast to sildenafil, which acutely lowered RV and arterial systolic pressure, pitavastatin-NP did not show acute hemodynamic effects (Figure 13). Collectively, these results suggested that pitavastatin-NP had a mechanism distinct from vasodilation in attenuating the remodel- ing of the pulmonary arteries.

Effect of pitavastatin-NP on the survival rate of rats with MCT-induced PAH: Finally, we examined the effect of pitavastatin-NP on the survival rate of rats with MCTinduced $\mathrm{PAH}$ on day 35. Compared with treatment with vehicle, FITC-NP, or sildenafil, treatment with pitavastatin-NP significantly improved the survival rate: $18 \%, 25 \%, 30 \%$, and $61 \%$ in the vehicle- $(n=34)$, sildenafil- $(n=28)$, FITC-NP- $(n=37)$, and pitavastatinNP-treated groups $(n=23)$, respectively (Figure 14).

\section{Discussion}

The major findings in this study are as follows: (1) FITC-NP was selectively delivered to the small pulmonary arteries and inflammatory cells in the lung after intravenous administration in a rat model of MCT-induced PAH; (2) pitavastatin-NP attenuated the progression of established PAH and improved survival; and (3) the beneficial effects of pitavastatin-NP were associated with antiinflammatory and anti-proliferative effects, which were mechanisms distinct from those of the PDE5 inhibitor, sildenafil.

PLGA-NP is known to distribute and accumulate in tissues with enhanced vascular permeability after intravenous administration. ${ }^{17,35)}$ As vascular permeability is enhanced in $\mathrm{PAH},{ }^{36)}$ intravenous PLGA-NP is probably delivered to the pulmonary vascular wall by passive targeting. In the present study, significant FITC signals were noted in the SMA-positive small pulmonary artery walls after the daily administration of FITC-NP. Additionally, $\mathrm{NP}$ was found to be incorporated into CD11b-positive inflammatory cells in the peripheral blood, lung, and BALF. As alveolar macrophages in the BALF are known to be derived largely from circulating monocytes, ${ }^{37)}$ these data suggested that intravenously administered PLGA-NP was incorporated by circulating monocytes and delivered to the alveolar space in the lung. These results indicated the benefit of intravenously administered PLGA-NP as a promising DDS in PAH.

Inflammation has been recently considered as a key pathophysiological factor that contributes to remodeling in $\mathrm{PAH}^{2)}$ Infiltrates of inflammatory cells to the lung and also inflammatory cytokines mediate the pathological features in patients with PAH. ${ }^{38-41)}$ For example, IL-6 is reported to promote the development and progression of pulmonary vascular remodeling by activating antiapoptotic and pro-proliferative pathways. ${ }^{41)}$ Although several anti-inflammatory therapies, such as dexamethasone, ${ }^{42)}$ FK506, ${ }^{43)}$ IL-1 receptor antagonist, ${ }^{44)}$ leukotriene $\mathrm{A}_{4}$ hydrolase, ${ }^{45}$ or CCR5 antagonist, ${ }^{46}$ ) have been investigated in animals, none of them have been clinically approved for the treatment of PAH. MCP-1 and its receptor CCR2 pathway, a key regulator of monocyte recruitment, have been shown to be involved in the pathogenesis of inflammation in cardiovascular diseases, ${ }^{47,48}$ including PAH. Plasma and lung tissue MCP-1 levels are reported to be increased in patients with $\mathrm{PAH},{ }^{40,49)}$ which decreased after treatment with epoprostenol. ${ }^{50)}$ In addition, treatment with

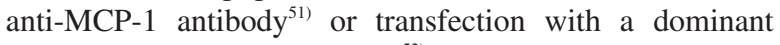
negative inhibitor of $\mathrm{MCP}-1^{52)}$ attenuated MCT-induced 

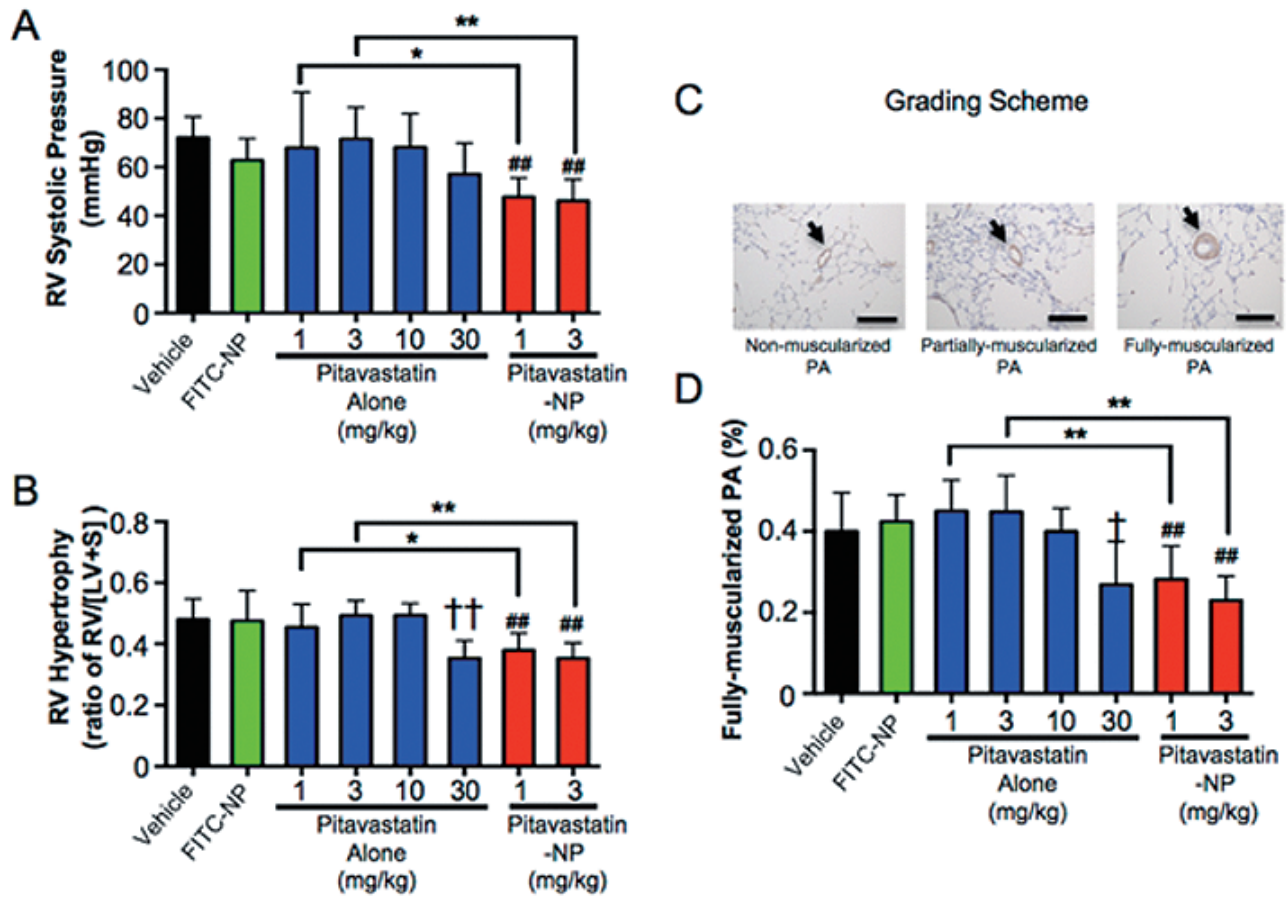

Figure 8. Effects of FITC-NP, pitavastatin, and pitavastatin-NP on RVSP, RVH, and muscularization of small pulmonary arteries. A: RVSP in the 8 experimental groups 3 weeks after the MCT injection. $n=8-10$ each. B: RVH assessed by the ratio of the weight of RV/[left ventricle (LV) + septum (S) ] in the 8 experimental groups 3 weeks after the MCT injection. $n=8-10$ each. C: Representative microscopic views of immunostaining with $\alpha$-SMA of the lung, and non-muscularized, partially muscularized, and fully muscularized pulmonary arteries. Scale bar: $50 \mu \mathrm{m}$. D: Percentage of fully muscularized pulmonary arteries in the 8 experimental groups. $n=8-10$ each. $* P<0.05, * * P<0.01$ versus the same dose of pitavastatin-alone and pitavastatin-NP groups. ${ }^{\#} P<0.01$ versus FITC-NP group. ${ }^{\dagger} P<0.05$, ${ }^{\dagger} P<0.01$ versus vehicle group.

$\mathrm{PAH}$ in the MCT-induced PAH rat model. These results showed that monocyte-mediated inflammation mediated by the MCP-1/CCR2 pathway can be a promising target in the treatment of PAH.

Statins are known to inhibit the activation of NF- $\kappa B$ and directly activate Akt, which are both key regulators of MCP-1. ${ }^{53,54)}$ Previously, we showed that pitavastatin-NP inhibited the MCP-1 induced chemotaxis of monocytes in vitro $^{18)}$ and also inhibited the expression of MCP-1 in the ischemic myocardium of the ischemia-reperfusion injury rat model in vivo ${ }^{17)}$ compared with pitavastatin alone. We also observed that the cardioprotective effect of pitavastatin-NP on ischemia-reperfusion injury in wildtype mice was blunted in CCR2-deficient mice (unpublished observation in our laboratory). In the present study, pitavastatin-NP significantly reduced the infiltration of CD68-positive monocytes/macrophages in the lungs of rats with MCT-induced PAH. Additionally, the result of the multiplex immunoassay suggested that pitavastatin-NP attenuated the elevation of cytokines/chemokines associated with monocytes/macrophages induced by MCT. Additionally, the fact that cytokines/chemokines associated with lymphocytes were significantly downregulated supports previous reports that showed that lymphocyte accumulation, such as $\mathrm{T}$ cells and $\mathrm{NK}$ cells, is attenuated in the lungs of MCT-induced PAH rats. ${ }^{55,56)}$ Collectively, these results suggest that the therapeutic effect of pitavastatin-NP was mediated by inhibiting monocytemediated inflammation by blocking the MCP-1/CCR2 pathway in the lung. Some subtypes of PAH, such as systemic sclerosis-associated PAH, are known to be strongly associated with monocyte-mediated inflammation. ${ }^{56)}$ However, as dysregulated immunity is a key pathophysiological factor of all PAH subtypes, ${ }^{2)}$ the anti-inflammatory effect of pitavastatin-NP deserves to be tested in any subtype of PAH.

Currently approved drugs for the treatment of $\mathrm{PAH}$ (PDE5 inhibitors, endothelin receptor antagonists, prostacyclins, etc.) are thought to deliver their therapeutic effects mainly through vasodilating activities, but not through direct anti-inflammatory and anti-proliferative effects. $^{2)}$ In contrast, statins are known to inhibit the proliferation of vascular SMCs down-regulating Rho, which leads to a reduction in MAPK activity, a strong regulator of SMC proliferation. ${ }^{57)}$ Statins also induce vasodilatory effects in some settings by the secondary activation of eNOS and prostacyclin synthase systems by downregulating Rho. ${ }^{8,58)}$ Previously, we showed that pitavastatin-NP directly inhibited the FBS-induced proliferation of human endothelial cells and pulmonary artery 

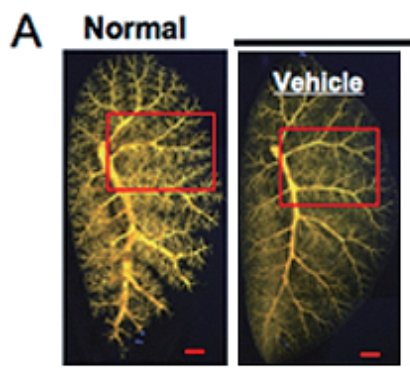

MCT+
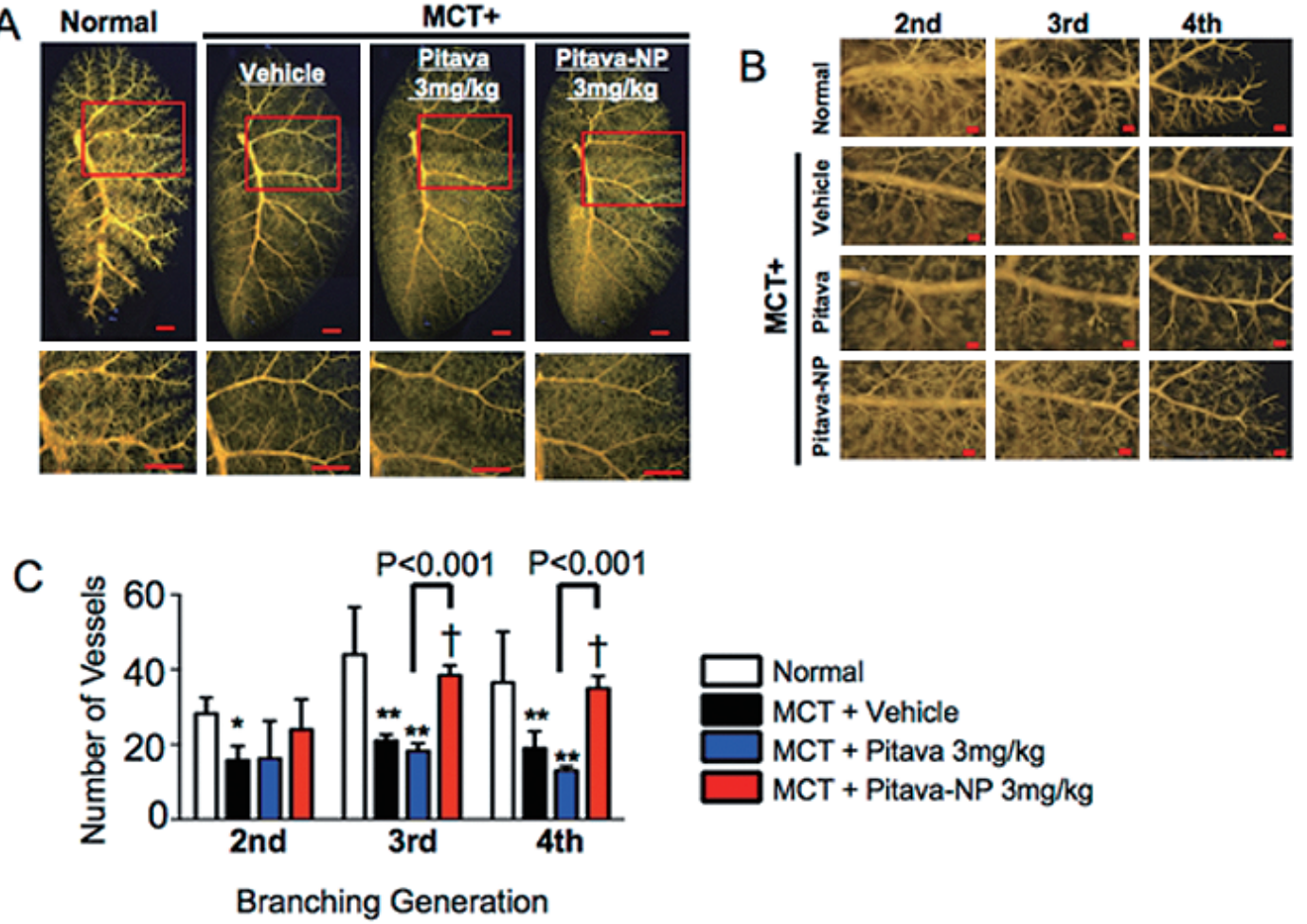

Figure 9. Effects of pitavastatin and pitavastatin-NP on the development of stenosis and obstruction in small pulmonary arteries 3 weeks after the MCT injection. A: Representative photographs of pulmonary artery angiography by Microfil. Scale bar: $1 \mathrm{~mm}$. B: Expanded views of the 2nd, 3rd, and 4th branches of the main pulmonary artery. C: Bar graph indicating the number of patent vessels branching from the 2 nd-, 3rd-, and 4th-generation branches, counted manually in the 4 experimental groups. Scale bar: $100 \mu \mathrm{m} . * P<0.05, * * P<0.01$ versus the normal group. ${ }^{\dagger} P<0.01$ versus the MCT + vehicle group. $n=4$ each.

SMCs. ${ }^{20)}$ In our short-term treatment protocol shown in Figure 3, intravenous pitavastatin-NP had no acute effects on RVSP, but treatment with the PDE5 inhibitor sildenafil decreased the elevated RVSP in the MCT-induced PAH model. Although we did not examine whether treatment with pitavastatin-NP activates eNOS or prostacyclin synthase systems in the chronic phases, these data suggest that the mechanism of pitavastatin-NP (mainly antiinflammatory and anti-proliferative effects) in ameliorating MCT-induced PAH is different from that of sildenafil (vasodilating actions).

Clinical trials of statins that failed to show clinical benefits in patients with PAH suggested that the oral administration of statins at clinically approved doses appears to be insufficient to exert therapeutic effects. ${ }^{14,15)}$ On the other hand, the therapeutic dose of statins in animal studies that showed its beneficial effects in PAH was relatively high (simvastatin 2-20 mg/kg/day, fluvastatin $1 \mathrm{mg} / \mathrm{kg} /$ day, or rosvastatin $2-10 \mathrm{mg} / \mathrm{kg} /$ day). ${ }^{9-13)}$ Since the oral administration of high-dose statins in humans is known to be harmful due to serious side effects, such as rhabdomyolysis, selective DDS with intravenous PLGA-NP can reduce the total amount of statin required. Indeed, in the present study, $1 \mathrm{mg} / \mathrm{kg}$ of pitavastatin-NP was equivalent to 30 $\mathrm{mg} / \mathrm{kg}$ of pitavastatin alone relative to the reduction of
RVSP. We have confirmed the safety limit of pitavastatin$\mathrm{NP}$ in rats as $2 \mathrm{mg} / \mathrm{kg}$ (unpublished data in our laboratory), suggesting that its intravenous administration at the dose of $1 \mathrm{mg} / \mathrm{kg}$ can be safe and feasible. However, as these data are derived only from rodent models, it is difficult to estimate the appropriate doses of pitavastatin-NP for patients with PAH. Thus, phase II clinical trials are needed to confirm the efficacy and safety of pitavastatinNP for PAH in humans.

In summary, nanoparticle-mediated targeting of pitavastatin to the small pulmonary arteries and inflammatory cells by intravenous administration attenuated the progression of established PAH and improved the survival in the MCT-PAH model, which was associated with antiinflammatory and anti-proliferative effects. We have completed the phase I clinical trial on intravenously administered pitavastatin-NP ( UMIN 000014940, UMIN 000019189) and confirmed the safety and tolerability in healthy subjects; ${ }^{59)}$ a phase II clinical trial for patients with $\mathrm{PAH}$ is now under contemplation. Pitavastatin-NP can be developed as a novel category of therapeutics for PAH. 

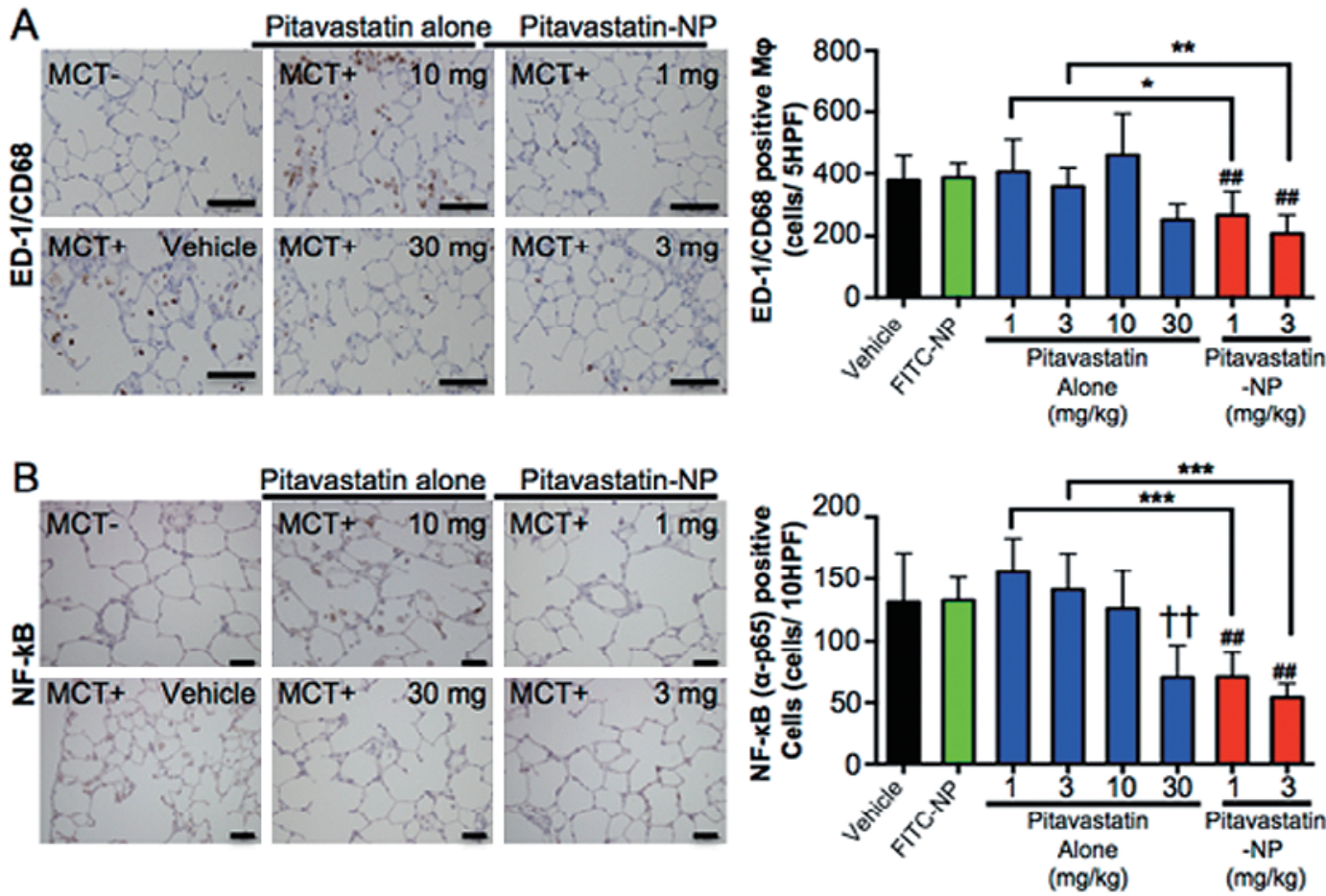

Figure 10. Effects of pitavastatin and pitavastatin-NP on the infiltration of monocytes and NF- $\mathrm{KB}$ activation 3 weeks after the MCT injection. A: Immunostaining with CD68 of lungs from rats in the 8 experimental groups. The bar graph indicates the number of CD68-positive monocytes per 5 high-power fields (HPFs) in each group. Scale bar: $50 \mu \mathrm{m} . n=8-10$ each. B: Immunostaining with NF- $\mathrm{KB}(\alpha-\mathrm{p} 65)$ of lungs from rats in the 8 experimental groups. The bar graph indicates the number of NF$\kappa \mathrm{B}(\alpha-$-p65) -positive cells per $10 \mathrm{HPFs}$ in each group. Scale bar: $50 \mu \mathrm{m} . n=8-10$ each. $* P<0.05, * * P<0.01, * * * P<0.001$ versus pitavastatin-alone and pitavastatin-NP groups with the same dose. ${ }^{\# \#} P<0.01$ versus the FITC-NP group. ${ }^{\dagger} P<0.05,{ }^{\dagger \dagger} P<$ 0.01 versus the vehicle group.

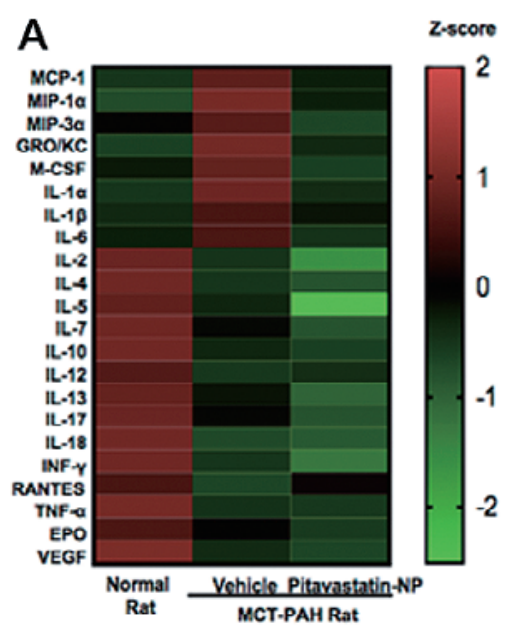

B

Figure 11. Cytokine/chemokine levels in the lung measured by multiplex immunoassay. A: Heat map showing the means of the z-scores of the expression of cytokines/chemokines in the lungs of normal rats and MCT-induced PAH rats treated with the vehicle of pitavastatin-NP. The Z-score was calculated as $\mathrm{z}=(\mathrm{x}-\mathrm{mean}) / \mathrm{SD}$. B: Table showing the levels of cytokines/chemokines in the lungs of normal rats and MCT-induced PAH rats treated with the vehicle of pitavastatin-NP. $P$ values shown in the table are versus the normal rat group. $* P<0.05$, $* * P<0.01$ versus the vehicle group. 


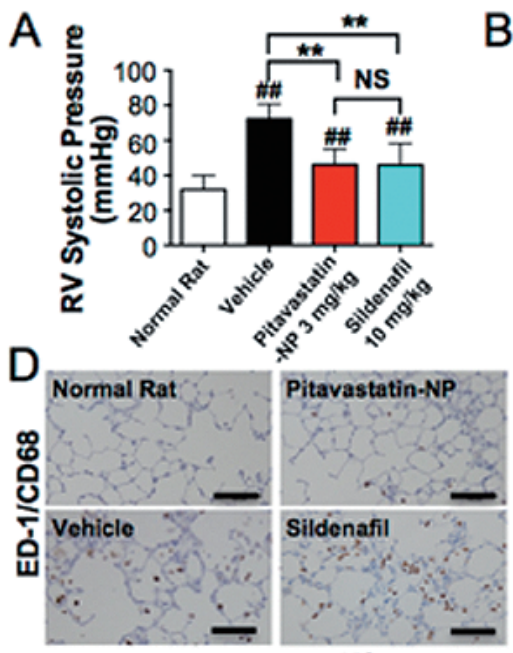

NS

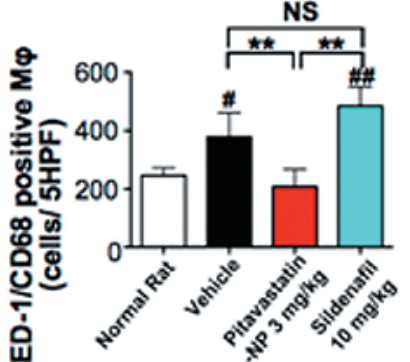

B
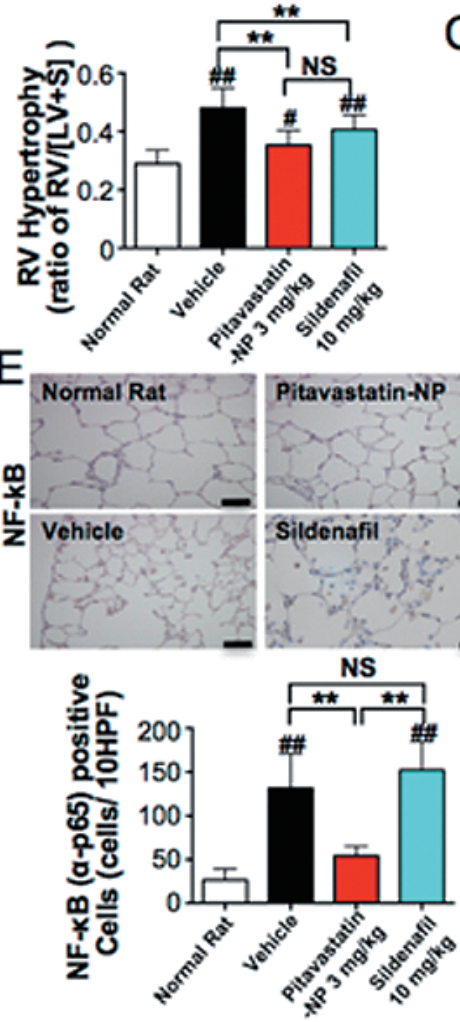

C

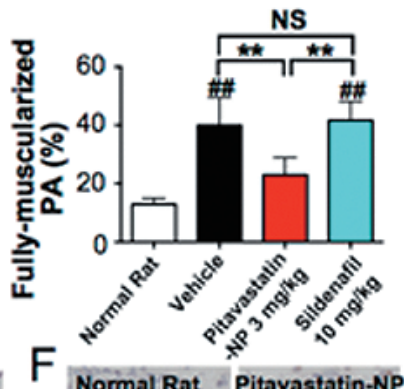

Figure 12. Comparisons of the effects of pitavastatin-NP and sildenafil on MCT-induced PAH. A: RVSP in the 4 experimental groups 3 weeks after the MCT injection. $n=8-10$ each. B: RVH assessed as the ratio of the weight of RV/[left ventricle (LV) + septum (S) ] in the 4 experimental groups 3 weeks after the MCT injection. $n=8-10$ each. C: Remodeling of the small pulmonary arteries assessed as the percentage of fully muscularized pulmonary arteries in the 4 experimental groups 3 weeks after the MCT injection. $n=8-10$ each. D: Immunostaining with CD68 of lungs from rats in the 4 experimental groups. The bar graph indicates the number of CD68-positive monocytes per 5 high-power fields (HPFs) in each group. Scale bar: $50 \mu \mathrm{m} . n=8-10$ each. E: Immunostaining with NF- $\mathrm{BB}(\alpha-\mathrm{p} 65)$ of lungs from rats in the 4 experimental groups. The bar graph indicates the number of NF- $\kappa \mathrm{B}(\alpha-\mathrm{p} 65)$ -positive cells per $10 \mathrm{HPFs}$ in each group. Scale bar: $50 \mu \mathrm{m} . n=8-10$ each. F: Immunostaining with PCNA of lungs from rats in the 4 experimental groups. The bar graph indicates the ratio of the number of PCNA-positive cells/total numbers of vascular cells in each groups Scale bar: $50 \mu \mathrm{m}$. ${ }^{* *} P<0.01$ versus the vehicle group. ${ }^{\#} P<0.05,{ }^{\#} P<0.01$ versus the normal rat group. $n=8$-10 each.
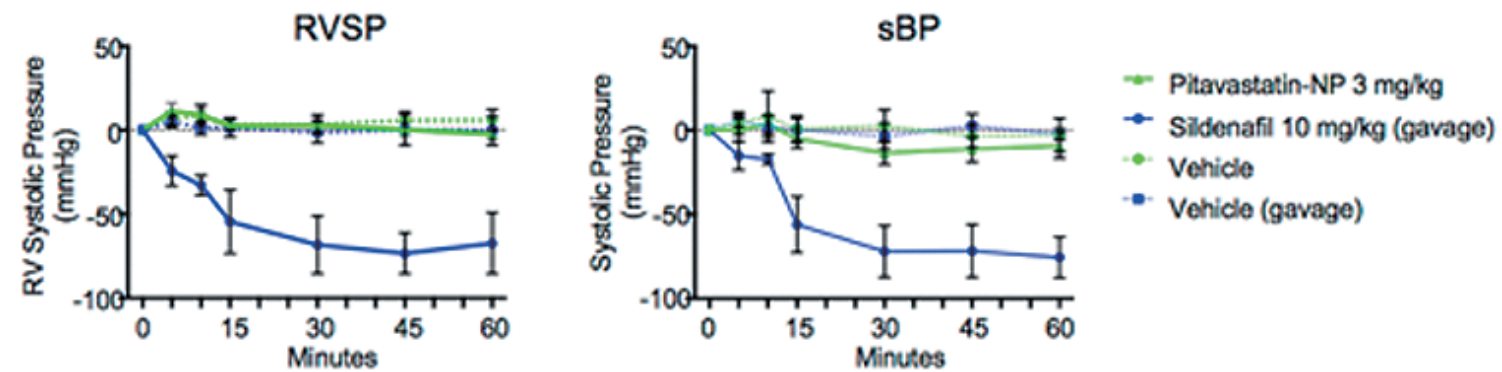

Figure 13. Acute hemodynamic effects of pitavastatin-NP and sildenafil in MCT-induced PAH rats. Results of continuous hemodynamic measurements. Changes in RV systolic pressure (RVSP) and arterial systolic pressure (sAP) from baseline were plotted for 1 hour. $n=3-4$ each. 

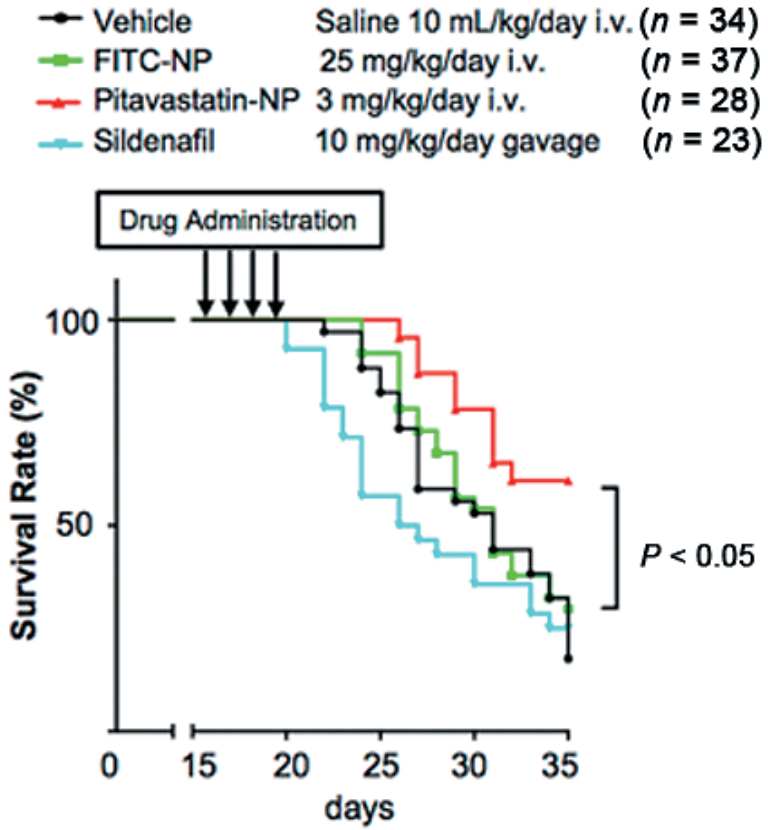

Figure 14. Effects of pitavastatin-NP and sildenafil on the survival rate. Survival curves analyzed by the Kaplan-Meier method in vehicle-, sildenafil-, FITC-NP-, and pitavastatin-NP-treated groups, respectively.

\section{Disclosures}

Conflicts of interest: Dr. Egashira holds a patent on the results reported in the present study. Dr. Tsutsui reports receiving grant supports from Astellas Pharma and Daiichi-Sankyo, lecture fees from Astellas Pharma, Otuka Pharmaceuticals, Takeda Pharmaceuticals, Daiichi-Sankyo, Tanabe-Mitsubishi Pharma, Boeringer Ingelheim Oharmaceuticals, Nocartis and Bayer. The remaining authors report no conflicts of interest.

\section{References}

1. Benza RL, Miller DP, Barst RJ, Badesch DB, Frost AE, McGoon MD. An evaluation of long-term survival from time of diagnosis in pulmonary arterial hypertension from the REVEAL Registry. Chest 2012; 142: 448-56.

2. Rabinovitch M, Guignabert C, Humbert M, Nicolls MR. Inflammation and immunity in the pathogenesis of pulmonary arterial hypertension. Circ Res 2014; 115: 165-75.

3. Jakóbisiak M, Bruno S, Skierski JS, Darzynkiewicz Z. Cell cycle-specific effects of lovastatin. Proc Natl Acad Sci U S A 1991; 88: 3628-32.

4. Laufs U, Liao JK. Post-transcriptional regulation of endothelial nitric oxide synthase mRNA stability by Rho GTPase. J Biol Chem 1998; 273: 24266-71.

5. Yoshida M, Sawada T, Ishii H, et al. HMG-CoA reductase inhibitor modulates monocyte-endothelial cell interaction under physiological flow conditions in vitro: involvement of Rho GTPase-dependent mechanism. Arterioscler Thromb Vasc Biol 2001; 21: 1165-71.

6. Sundaresan M, Yu ZX, Ferrans VJ, et al. Regulation of reactiveoxygen-species generation in fibroblasts by Rac1. Biochem J
1996; 318: 379-82.

7. Takemoto M, Liao JK. Pleiotropic effects of 3-hydroxy-3methylglutaryl coenzyme A reductase inhibitors. Arterioscler Thromb Vasc Biol 2001; 21: 1712-9.

8. Wolfrum S, Jensen KS, Liao JK. Endothelium-dependent effects of statins. Arterioscler Thromb Vasc Biol 2003; 23: 729-36.

9. Nishimura T, Faul JL, Berry GJ, et al. Simvastatin attenuates smooth muscle neointimal proliferation and pulmonary hypertension in rats. Am J Respir Crit Care Med 2002; 166: 1403-8.

10. Nishimura T, Vaszar LT, Faul JL, et al. Simvastatin rescues rats from fatal pulmonary hypertension by inducing apoptosis of neointimal smooth muscle cells. Circulation 2003; 108: 1640-5.

11. Murata T, Kinoshita K, Hori M, et al. Statin protects endothelial nitric oxide synthase activity in hypoxia-induced pulmonary hypertension. Arterioscler Thromb Vasc Biol 2005; 25: 2335-42.

12. Girgis RE, Mozammel S, Champion HC, et al. Regression of chronic hypoxic pulmonary hypertension by simvastatin. Am J Physiol Lung Cell Mol Physiol 2007; 292: L1105-10.

13. Li XL, Guan RJ, Li JJ. Attenuation of monocrotaline-induced pulmonary arterial hypertension in rats by rosuvastatin. J Cardiovasc Pharmacol 2012; 60: 219-26.

14. Wilkins MR, Ali O, Bradlow W, et al. Simvastatin as a treatment for pulmonary hypertension trial. Am J Respir Crit Care Med 2010; 181: 1106-13.

15. Zeng WJ, Xiong CM, Zhao L, et al. Atorvastatin. Eur Respir J 2012; 40: 67-74.

16. Ichimura K, Matoba T, Nakano K, et al. A translational study of a new therapeutic approach for acute myocardial infarction: nanoparticle-mediated delivery of pitavastatin into reperfused myocardium reduces ischemia-reperfusion injury in a preclinical porcine model. PLoS One 2016; 11: e0162425.

17. Nagaoka K, Matoba T, Mao Y, et al. A new therapeutic modality for acute myocardial infarction: nanoparticle-mediated delivery of pitavastatin induces cardioprotection from ischemiareperfusion injury via activation of PI3K/Akt pathway and antiinflammation in a rat model. PLoS One 2015; 10: e0132451.

18. Katsuki S, Matoba T, Nakashiro S, et al. Nanoparticle-mediated delivery of pitavastatin inhibits atherosclerotic plaque destabilization/rupture in mice by regulating the recruitment of inflammatory monocytes. Circulation 2014; 129: 896-906.

19. Tsukie N, Nakano K, Matoba T, et al. Pitavastatin-incorporated nanoparticle-eluting stents attenuate in-stent stenosis without delayed endothelial healing effects in a porcine coronary artery model. J Atheroscler Thromb 2013; 20: 32-45.

20. Chen L, Nakano K, Kimura S, et al. Nanoparticle-mediated delivery of pitavastatin into lungs ameliorates the development and induces regression of monocrotaline-induced pulmonary artery hypertension. Hypertension 2011; 57: 343-50.

21. Oda S, Nagahama R, Nakano K, et al. Nanoparticle-mediated endothelial cell-selective delivery of pitavastatin induces functional collateral arteries (therapeutic arteriogenesis) in a rabbit model of chronic hind limb ischemia. J Vasc Surg 2010; 52: 412-20.

22. Kubo M, Egashira K, Inoue T, et al. Therapeutic neovascularization by nanotechnology-mediated cell-selective delivery of pitavastatin into the vascular endothelium. Arterioscler Thromb Vasc Biol 2009; 29: 796-801.

23. Nakashiro S, Matoba $\mathrm{T}$, Umezu $\mathrm{R}$, et al. Pioglitazoneincorporated nanoparticles prevent plaque destabilization and rupture by regulating monocyte/macrophage differentiation in ApoE-/- mice. Arterioscler Thromb Vasc Biol 2016; 36: 491500 .

24. Mao Y, Koga JI, Tokutome M, et al. Nanoparticle-mediated delivery of pitavastatin to monocytes/macrophages inhibits left ventricular remodeling after acute myocardial infarction by inhibiting monocyte-mediated inflammation. Int Heart J 2017; 58: 615-23.

25. Ishikita A, Matoba T, Ikeda G, et al. Nanoparticle-mediated delivery of mitochondrial division Inhibitor 1 to the myocardium protects the heart from ischemia-reperfusion injury through inhi- 
bition of mitochondria outer membrane permeabilization: A new therapeutic modality for acute myocardial infarction. J Am Heart Assoc 2016; 5.

26. Nakano Y, Matoba T, Tokutome M, et al. Nanoparticle-mediated delivery of irbesartan induces cardioprotection from myocardial ischemia-reperfusion injury by antagonizing monocyte-mediated inflammation. Sci Rep 2016; 6: 29601.

27. Ikeda G, Matoba T, Nakano Y, et al. Nanoparticle-mediated targeting of cyclosporine A enhances cardioprotection against ischemia-reperfusion injury through inhibition of mitochondrial permeability transition pore opening. Sci Rep 2016; 6: 20467.

28. Nagahama R, Matoba T, Nakano K, Kim-Mitsuyama S, Sunagawa K, Egashira K. Nanoparticle-mediated delivery of pioglitazone enhances therapeutic neovascularization in a murine model of hindlimb ischemia. Arterioscler Thromb Vasc Biol 2012; 32: 2427-34.

29. Masuda S, Nakano K, Funakoshi K, et al. Imatinib mesylateincorporated nanoparticle-eluting stent attenuates in-stent neointimal formation in porcine coronary arteries. J Atheroscler Thromb 2011; 18: 1043-53.

30. Kimura S, Egashira K, Nakano K, et al. Local delivery of imatinib mesylate (STI571)-incorporated nanoparticle ex vivo suppresses vein graft neointima formation. Circulation 2008; 118: S65-70.

31. Kimura S, Egashira K, Chen L, et al. Nanoparticle-mediated delivery of nuclear factor kappaB decoy into lungs ameliorates monocrotaline-induced pulmonary arterial hypertension. Hypertension 2009; 53: 877-83.

32. Handoko ML, de Man FS, Happé CM, et al. Opposite effects of training in rats with stable and progressive pulmonary hypertension. Circulation 2009; 120: 42-9.

33. Razavi H, Dusch MN, Zarafshar SY, Taylor CA, Feinstein JA. A method for quantitative characterization of growth in the 3-D structure of rat pulmonary arteries. Microvasc Res 2012; 83: 146-53.

34. Kido M, Du L, Sullivan CC, Deutsch R, Jamieson SW. Thistlethwaite PA. Gene transfer of a TIE2 receptor antagonist prevents pulmonary hypertension in rodents. J Thorac Cardiovasc Surg 2005; 129: 268-76.

35. Danhier F, Ansorena E, Silva JM, Coco R, Le Breton A, Préat V. PLGA-based nanoparticles: an overview of biomedical applications. J Control Release 2012; 161: 505-22.

36. Ishihara T, Hayashi E, Yamamoto $\mathrm{S}$, et al. Encapsulation of Beraprost sodium in nanoparticles: analysis of sustained release properties, targeting abilities and pharmacological activities in animal models of pulmonary arterial hypertension. J Control Release 2015; 197: 97-104.

37. Cai Y, Sugimoto C, Arainga M, Alvarez X, Didier ES, Kuroda MJ. In vivo characterization of alveolar and interstitial lung macrophages in rhesus macaques: implications for understanding lung disease in humans. J Immunol 2014; 192: 2821-9.

38. Tuder RM, Groves B, Badesch DB, Voelkel NF. Exuberant endothelial cell growth and elements of inflammation are present in plexiform lesions of pulmonary hypertension. Am J Pathol 1994; 144: 275-85.

39. Pinto RF, Higuchi Mde L, Aiello VD. Decreased numbers of Tlymphocytes and predominance of recently recruited macrophages in the walls of peripheral pulmonary arteries from 26 patients with pulmonary hypertension secondary to congenital cardiac shunts. Cardiovasc Pathol 2004; 13: 268-75.

40. Sanchez O, Marcos E, Perros F, et al. Role of endotheliumderived $\mathrm{CC}$ chemokine ligand 2 in idiopathic pulmonary arterial hypertension. Am J Respir Crit Care Med 2007; 176: 1041-7.

41. Steiner MK, Syrkina OL, Kolliputi N, Mark EJ, Hales CA, Waxman AB. Interleukin-6 overexpression induces pulmonary hypertension. Circ Res 2009; 104: 236-44.

42. Price LC, Montani D, Tcherakian C, et al. Dexamethasone reverses monocrotaline-induced pulmonary arterial hypertension in rats. Eur Respir J 2011; 37: 813-22.

43. Spiekerkoetter E, Tian X, Cai J, et al. FK506 activates BMPR2, rescues endothelial dysfunction, and reverses pulmonary hypertension. J Clin Invest 2013; 123: 3600-13.

44. Voelkel NF, Tuder RM, Bridges J, Arend WP. Interleukin-1 receptor antagonist treatment reduces pulmonary hypertension generated in rats by monocrotaline. Am J Respir Cell Mol Biol 1994; 11: 664-75.

45. Tian W, Jiang X, Tamosiuniene R, et al. Blocking macrophage leukotriene b4 prevents endothelial injury and reverses pulmonary hypertension. Sci Transl Med 2013; 5: 200ra117.

46. Amsellem V, Lipskaia L, Abid S, et al. CCR5 as a treatment target in pulmonary arterial hypertension. Circulation 2014; 130: 880-91.

47. Egashira K. Molecular mechanisms mediating inflammation in vascular disease: special reference to monocyte chemoattractant protein-1. Hypertension 2003; 41: 834-41.

48. Matoba T, Egashira K. Anti-inflammatory gene therapy for cardiovascular disease. Curr Gene Ther 2011; 11: 442-6.

49. Itoh $\mathrm{T}$, Nagaya $\mathrm{N}$, Ishibashi-Ueda $\mathrm{H}$, et al. Increased plasma monocyte chemoattractant protein-1 level in idiopathic pulmonary arterial hypertension. Respirology 2006; 11: 158-63.

50. Katsushi H, Kazufumi N, Hideki F, et al. Epoprostenol therapy decreases elevated circulating levels of monocyte chemoattractant protein-1 in patients with primary pulmonary hypertension. Circ J 2004; 68: 227-31.

51. Kimura H, Kasahara Y, Kurosu K, et al. Alleviation of monocrotaline-induced pulmonary hypertension by antibodies to monocyte chemotactic and activating factor/monocyte chemoattractant protein-1. Lab Invest 1998; 78: 571-81.

52. Ikeda Y, Yonemitsu Y, Kataoka C, et al. Anti-monocyte chemoattractant protein-1 gene therapy attenuates pulmonary hypertension in rats. Am J Physiol Heart Circ Physiol 2002; 283: H2021-8.

53. Ortego M, Bustos C, Hernández-Presa MA, et al. Atorvastatin reduces NF-kappaB activation and chemokine expression in vascular smooth muscle cells and mononuclear cells. Atherosclerosis 1999; 147: 253-61.

54. Kureishi Y, Luo Z, Shiojima I, et al. The HMG-CoA reductase inhibitor simvastatin activates the protein kinase Akt and promotes angiogenesis in normocholesterolemic animals. Nat Med 2000; 6: 1004-10.

55. Ormiston ML, Deng Y, Rundle N, et al. A lymphocytedependent mode of action for imatinib mesylate in experimental pulmonary hypertension. Am J Pathol 2013; 182: 1541-51.

56. Ormiston ML, Chang C, Long LL, et al. Impaired natural killer cell phenotype and function in idiopathic and heritable pulmonary arterial hypertension. Circulation 2012; 126: 1099-109.

57. Christmann RB, Hayes E, Pendergrass S, et al. Interferon and alternative activation of monocyte/macrophages in systemic sclerosis-associated pulmonary arterial hypertension. Arthritis Rheum 2011; 63: 1718-28.

58. Kamiyama M, Utsunomiya K, Taniguchi K, et al. Contribution of Rho A and Rho kinase to platelet-derived growth factor-BBinduced proliferation of vascular smooth muscle cells. J Atheroscler Thromb 2003; 10: 117-23.

59. Nakano K, Matoba T, Koga J, et al. Safety, Tolerability, and Pharmacokinetics of NK-104-NP:A Multicenter, Randomized, Placebo Controlled Phase I Investigator-Initiated Trial for intravenous Administration of pitavastatin-loaded PLGA nanoparticles (NK-104-NP) in Healthy Japanese Male Subjects. Int Heart J 2018; 59: 1015-25. 